\title{
Jean Clédière
}

\section{F. D. Schubart et la Révolution Française}

Ecrivain wurtembergeois, né à Obersontheim en 1739, mort à Stuttgart en octobre 1791, Schubart est aujourd'hui connu à un triple titre: comme poète, en raison des épreuves qui ont marqué son existence, et comme journaliste. Le poète, dont l'œuvre se partage entre le Volkslied et des compositions qui rappellent Klopstock, ne retiendra pas ici notre attention. L'homme est surtout célèbre pour avoir passé dix années de sa vie à la prison du Hohenasperg, près de Ludwigsburg; bien que le duc CharlesEugène de Wurtemberg qui le fit arrêter n'ait jamais justifié cette sanction, celle-ci n'est probablement pas sans rapport avec le troisième aspect, à notre avis le plus original, présenté par cet auteur: son activité de journaliste ${ }^{1}$. On peut en effet penser que c'est pour avoir commis quelque impertinence majeure que Schubart fut condamné à la détention qui le frappa en pleine maturité et à laquelle il ne survécut que quelques années.

En 1774, à Augsbourg, Schubart avait fondé la Deutsche Cbronik, un périodique comme il en existait tant dans les pays allemands à cette époque. Il s'était fixé pour but de former ses lecteurs en les informant; pour cela, deux fois par semaine, il les tenait au courant de l'actualité politique et littéraire, ces deux rubriques étant représentées à parts à peu près égales. A travers ces informations et les commentaires dont il les accompagnait, Schubart entendait répandre parmi ses compatriotes un double patriotisme: il voulait les rendre fiers d'être Souabes et d'être Allemands à la fois ${ }^{2}$. C'est dire que la polémique antifrançaise inspire le plus souvent ses jugements, prenant fréquemment le pas sur les critères esthétiques. Durant les quatre années qui précédèrent son arrestation, Schubart se passionna et tenta de passionner ses lecteurs pour ce qu'il considérait être les grandes causes de la liberté: la Pologne, dont s'opère le premier partage, mais surtout la guerre d'Indépendance des Etats-Unis. Schubart, ces années-là, fit une large part aux événements d'outre-Atlantique. Comme nombre d'intellectuels allemands, il épousa chaleureusement la cause de ceux qu'on appelait alors les Insur-

${ }^{1}$ Cf. par ex. Erich Scbairer, C. F. D. Schubart als politischer Journalist (Tübingen 1914) et Horst Adamietz, C. F. D. Schubarts Volksblatt „Deutsche Chronik“" (Berlin 1943).

Schubart intitula sa revue Deutsche Chronik, puis Teutscbe Cbronik entre 1774 et 1777 . A partir de 1787, il lui donne pour titre Vaterländische Chronik, puis Vaterlandscbronik et enfin Cbronik tout court, signifiant par là l'importance des événements extérieurs, notamment la Révolution Française. Nous citons sous le titre simplifié Chronik.

${ }_{2}$ Cf. sur ce point, Gunter Volz, Schwabens streitbare Musen. Schwäbische Literatur des 18.Jahrhunderts im Wettstreit der deutschen Stāmme (Stuttgart 1986). 
gents $^{3}$. Il dénonça le "Soldatenhandel» par lequel nombre de princes allemands - dont le duc de Wurtemberg - vendaient leurs soldats aux autres puissances, notamment à l'Angleterré.

En mars 1777, l'éditeur de la Teutsche Chronik, tomba dans un guet-apens que lui avait fait tendre le duc, sans doute irrité, entre autres choses, d'être ainsi mis en cause. Dix années durant, Schubart sera incarcéré au Hohenasperg dans des conditions qui progressivement furent adoucies mais qui devaient le marquer à tout jamais. La cour de Prusse fut pour beaucoup dans sa libération qui survint en juin 1787: pas plus que son arrestation, elle ne reçut d'explication de la part du duc. Après cette coupure, Schubart, curieusement, put reprendre l'édition de la revue qu'il intitula dès lors $\mathrm{Va}$ terländische Cbronik avant d'en abréger le titre en Chronik tout court. Mais désormais celle-ci était imprimée sur les presses de la Karlsschule ${ }^{5}$, bénéficiant de ce qu'on appelait la «Zensurfreiheit : autrement dit, il devait se censurer lui-même. On comprendra qu'instruit par son propre exemple, Schubart ait été naturellement incité à la prudence. Il évite dès lors de mettre en cause son bourreau de la veille.

Ces quelques éléments biographiques ne sont pas là seulement pour situer Schubart. Ils suggèrent qu'une cassure aussi importante que celle introduite par l'arrestation arbitraire dont il fut victime et la détention de dix années qui s'en suivit n'ont pas été sans influer profondément sur la psychologie et le mode de pensée d'un homme qui, prématurément vieilli, a perdu quelque chose de son énergie et de sa virulence. Certes le ton de la Chronik, qui va reparaître de 1787 à 1791, ne diffère pas essentiellement de celui qui la distinguait quinze ans auparavant. Mais Schubart fait maintenant preuve d'une retenue qui n'avait pas toujours été dans sa manière. Il serait sans doute exagéré de dire que le rebelle s'était fait valet du prince qui l'aurait de nouveau châtié à la première incartade ${ }^{6}$. Sur bien des points cependant, Schubart adopte des positions fort éloignées de celles qu'il avait autrefois défendues: ainsi, l'admirateur de Frédéric II devient-il le zélateur des édits de Wöllner, le pourfendeur de la fausse Aufklärung. C'est après tout un moyen de renouer avec la polémique antifrançaise qui lui tient tant à cœur: voltairiens, les Français incarnent à ses yeux ce que les Lumières peuvent avoir de superficiel et d'antireligieux.

Or, moins de deux ans après la libération de Schubart, éclate la Révolution Française. Elle va amener Schubart à reconsidérer notablement l'image qu'il avait jusqu'alors tracée de ce pays. Car l'image de la France avait, dans la Chronik, toujours occupé une place importante. Schubart n'avait cessé de mettre ses lecteurs en garde contre la gallomanie qui sévissait en Allemagne. Mise en garde qui se doublait d'une peinture systématiquement négative du caractère français. Après comme avant 1787 ,

${ }^{3}$ La mise au point la plus récente est celle de Horst Dippel, Germany and the American Revolution 1770-1800 (Wiesbaden 1978).

${ }^{4}$ Cf. Friedrich Knapp, Der Soldatenhandel deutscher Fürsten nach Amerika (Berlin 1864, rééd. Munich 1984).

${ }^{5}$ Cf. Robert Uhland, Geschichte der hohen Karlsschule in Stuttgart (Stuttgart 1953); Heinrich Wagner, Geschichte der Hohen Carls-Schule (Würzburg 1858).

${ }^{6}$ Cf. Wilfried F. Schoeller, Schubart, Leben und Meinungen eines schwäbischen Rebellen (Berlin 1979) et l'introduction de Peter Härtling à C. F. D.'Scbubart, Gedichte, ausgewählt von Peter Härtling (Frankfurt/M. 1968). 
Schubart entend être un "patriote ${ }^{7}$. Au fond, il ne cesse de répéter ce que Klopstock dénonçait dans son poème "An die Überschätzer der Ausländer*. Sous l'influence des événements de 1789, la France, de repoussoir va-t-elle devenir modèle, ce qui signifierait que Schubart n'avait jamais visé qu'une certaine francomanie, celle qui était le fait des princes et des milieux de ceux qui étaient prêts à renier leur patrie allemande? La mutation, chez Schubart, ne fut à vrai dire, ni aussi subite, ni aussi radicale.

En effet, dès 1788 , le "patriote" qu'est Schubart semble déjà en passe de nuancer fortement ses jugements sur la France et les Français. Sans doute renoue-t-il encore à plusieurs reprises avec les clichés et les stéréotypes auxquels la Chronik nous avait de longue date habitués. Mais on voit poindre un renversement de la perspective qui se précisera à partir de 1789 . Parlant le 20 mai 1788 du vent de fronde parlementaire qui souffle aussi bien sur Paris que sur la province, Schubart note que l'esprit français est moins que par le passé prisonnier de la mode, de la frivolité et de la vanité. Fait significatif: Schubart donne désormais au mot *patriote" une acception étendue. Le patriote est maintenant celui qui s'oppose au despote. Défenseur de la liberté, le "patriote* s'oppose à l'arbitraire du prince ${ }^{8}$.

Or Schubart perçoit en France beaucoup de signes d'un mécontentement qui, si Louis XVI n'y prend pas garde, pourrait bien mener à la guerre civile. Sans doute la cible en est-elle les ministres plus que le roi lui-même. Mais c'est à ce dernier que va l'avertissement.

Pourtant Louis XVI est considéré par Schubart avec une bienveillance et une sympathie qui persisteront encore au moment de Varennes. Rappelons brièvement comment, presque quinze ans auparavant, Schubart avait relaté les premières déclarations et les premières initiatives de Louis XVI. Il en avait d'emblée retenu l'image d'un homme avant tout soucieux du bien de ses sujets: «Il veut, écrivait-il en 1775, faire du bien à ce peuple futile et frivole, même si celui-ci se dresse là-contre.» L'action de Louis XVI est, dans la Cbronik, décrite comme bénéfique: Louis XVI décrète des mesures charitables au profit des orphelinats et des hôpitaux. Mendicité, prostitution et vagabondage tendent à disparaître. Le roi encourage les sciences et l'agriculture. A plusieurs reprises, Schubart souligne la prospérité financière et économique du royaume de France. Au-delà des mesures concrètes prises par Louis XVI, Schubart insiste sur son souci d'instaurer plus d'équité dans les rapports sociaux. Le roi veut marquer sa sollicitude envers les petites gens, dût-il pour cela heurter la noblesse'. En juin 1776, la Chronik contient un long article consacré à la suppression de la corvée. En cela d'ailleurs, elle s'inspire étroitement de l'article paru sur le même sujet dans le Teutscher Merkur de Wieland. «Les édits de Louis XVI, écrit Schubart, sont des chefs-d'œuvre de

7 Cf. Cosmopolitisme, Patriotisme et Xénophobie en Europe au siècle des Lumières. Actes du colloque international (Strasbourg 2-5 octobre 1985) édités par Gontbier-Louis Fink (Strasbourg 1987) et Revue d'Allemagne (XVIII.1987.4).

${ }^{8}$ Chronik, 20 mai 1788, 329 sq.

9 Sur les débuts du règne de Louis XVI, cf. Chronik, 20 juin 1774, 187 sq., 30 mai 1776, 344 sq. -Dieses große und glückliche Reich erhebt sich zu einem Gipfel von Ansehen und Glückseligkeit ... et passim, juin, novembre 1776 . 
la raison, de la bonté, en même temps que de l'éloquence. Puisse seulement le roi se garder des flatteurs qui l'entourent! ${ }^{10}$

On pourra être un peu surpris de voir Schubart féliciter plus particulièrement Louis XVI d'avoir opéré un spectaculaire redressement militaire grâce à l'action de son ministre, le comte de Saint-Germain ${ }^{11}$. Or, il y a à cela une explication psychologique qui vaudra également lorsque Schubart saluera les premières initiatives de la Révolution. Les mesures prises par Saint-Germain vont en effet dans le sens des réformes qui rendent aux Français une énergie dont Schubart avait tendance à les tenir pour congénitalement dépourvus. «Saint-Germain, écrit-il, sort la France de sa léthargie. Grâce à lui, l'armée française ressemblera à une véritable armée et non plus à une collection de figurants chaussant bas de soie.»

Notons que Schubart, à l'aube du règne de Louis XVI, partage ici une euphorie telle que nous la décrit Charles Aubertin dans son livre sur l'Esprit public en France au XVIIIème siècle ${ }^{12}$. Il reprend, en l'amplifiant et en le simplifiant, ce qui est conforme à son style habituel de journaliste, ce que disent alors beaucoup de Français. Il lui plaît de voir les événements s'accorder à quelques-uns des cadres préétablis où il aime voir évoluer les hommes et se définir les régimes. Avec Louis XVI, il croit tenir un exemplaire quasi parfait du sbon roi*; et que Marie-Antoinette appartienne pour moitié à la Maison de Habsbourg lui est un motif supplémentaire de sympathie envers la famille royale.

Louis XVI est également admirable pour avoir promulgué l'édit de tolérance, en même temps d'ailleurs qu'il réintroduisait chez les officiers le respect de la religion ${ }^{\mathbf{1 3}}$. Pour toutes ces raisons, le roi de France n'est pas loin d'incarner aux yeux de Schubart cette Aufklärung à l'allemande, celle qui est synonyme de tolérance mais ne verse jamais dans l'irreligion.

Que sur ce fond d'euphorie vienne à éclater une émeute et Schubart l'attribue immédiatement à cet «esprit de sédition* qui s'empare régulièrement des hommes. Ainsi, loin de voir dans les troubles qui ont éclaté à Reims et à Paris l'indice d'un malaise plus profond, il ne veut y voir à l'œuvre que ce même «esprit de désordre. Il félicite Louis XVI d'y avoir fait face avec fermeté ${ }^{14}$. Et c'est avec quelque tristesse qu'il lui faut en conclure au relatif discrédit où est tombé le roi. Il évoque l'impertinente floraison de libelles que l'on observe à Paris en remarquant ironiquement que semblable phénomène ne risque pas de se produire chez les Allemands, tant ceux-ci sont respectueux de leurs souverains ${ }^{15}$.

10 Chronik, 30 mai $1776,344$.

11 Chronik, 24 novembre 1776, 743: «die verlorene Spann- und Schnellkraft der Nation wird nach und nach wieder hergestellt.*

${ }_{12}$ Charles Aubertin, L'esprit public en France au XVIIIè siècle (Paris 1889) et François Bluche, La vie quotidienne au temps de Louis XVI (Paris 1980).

${ }^{13}$ Chronik, 24 juin 1776, 404 et 22 février 1788, 124 sq. ainsi que 11 janvier 1788, 28. Schubart fustige l'opposition des parlementaires envers cet édit.

${ }_{14}$ Chronik, 29 mai 1775, 337 sq. *Der Geist des Aufruhrs ist auch in diese glücklichen Provinzen eingedrungen ...* ou encore: «Schon weit hat dieser Geist der Unruhe um sich gegriffen.* 15 Ibid., 438. 
Lorsque l'on évoque les répercussions de la Révolution Française en Allemagne du Sud, on a coutume de citer le Stift de Tübingen. Selon le témoignage de l'un des boursiers de cette institution, l'engouement aurait été général parmi ces jeunes intellectuels. Longtemps après encore, cet enthousiasme ne semblait pas s'être tari, en particulier parmi les pasteurs et les gens lettrés qui, ainsi que le notera un diplomate saxon chargé des affaires bavaroises, sont en partie atteints de cette manie ${ }^{16}$. L'anecdote, dont certains se demandent aujourd'hui si elle ne serait pas une légende, qui veut que trois des plus prestigieux pensionnaires du Stift, Hölderlin, Hegel et Schelling, aient planté un arbre de la liberté à l'annonce de la prise de la Bastille, est caractéristique de cet état d'esprit" ${ }^{17}$.

Mais celui-ci n'était pas répandu seulement parmi les séminaristes protestants. La Karlsschule ne semble pas avoir échappé à cet engouement. Certains élèves y constituèrent un club de la liberté qui se mit en relation avec les clubs de Strasbourg. On lisait les journaux français, strasbourgeois en particulier ${ }^{18}$. Dans ses Mémoires, J. Kerner témoigne de cette effervescence. Lui-même se rendit à Strasbourg pour observer de plus près la «renaissance de l'humanité», cependant que d'autres disciples se contentaient de proclamer leur sympathie sur place ${ }^{19}$.

De fait, ces manifestations ne furent que mollement réprimées par les autorités de l'Ecole caroline, sauf lorsque la renommée de l'établissement se trouvait mise en cause, par des caricatures par exemple. Parmi les professeurs, les prises de position furent plus discrètes, si l'on excepte le cas de C. F. Cotta, professeur de droit, qui fit également le voyage de Strasbourg. Face à cette poussée de fièvre révolutionnaire, il semble que Charles-Eugène ait réagi avec beaucoup de modération; par prudence, en raison de l'appartenance au Wurtemberg de villes comme Montbéliard et Riquewihr, il se garde tout d'abord de prendre ouvertement parti contre la Révolution. Son Journal de Voyage porte trace de cette attitude pour le moins ambiguë dont Charles-Eugène ne se départit pas, aussi longtemps que l'étincelle de la Révolution ne risquait pas d'atteindre l'Allemagne ${ }^{20}$.

Or, c'est sur les presses de la Karlsschule qu'était imprimée la Cbronik. Schubart, dans ce milieu, devait avoir connaissance des journaux strasbourgeois dans lesquels il ne fait pas mystère de puiser. Il pouvait le faire assez librement, n'ayant guère à craindre de la part de Charles-Eugène qu'il sanctionne cette liberté dès lors qu'elle ne le mettait pas directement en cause.

Comment Schubart était-il informé de ce qui se passait en France? La Cbronik fait à plusieurs reprises allusion à des correspondants parisiens ou strasbourgeois. Ceux-ci

${ }^{16}$ Cf. outre Jacques Droz, L'Allemagne et la Révolution Française (Paris 1950) 287 sq. Wilhelm Lang, Von und aus Schwaben, 1. Heft, 57 (Stuttgart 1885); Julius Hartmann, Schillers Jugendfreunde, (Stuttgart 1904) 80.

17 Cf. J. Noutary, La Souabe devant la Révolution Française in Le Wurtemberg (Tübingen 1950) 233-243.

${ }_{18}$ Cf. Ubland, 249 et Karl Pfaff, Geschichte Württembergs, Bd. 5 (Reutlingen 1820) 185.

19 Justinus Kerner, Das Bilderbuch aus meiner Knabenzeit. Erinnerungen aus den Jahren 1786 bis 1804 (Braunschweig 1849, rééd. Frankfurt/M. 1978) 45 sq.

${ }^{20} \mathrm{Cf}$. Herzog Carl Eugen von Württemberg, Tagebücher seiner Rayssen in den Jahren 1783-1792, hrsg. von Robert Ubland (Stuttgart 1968) 380 sq. 
existaient-ils réellement? On peut parfois en douter. Les lettres que publie Schubart ne sont peut-être qu'un subterfuge qui lui permet de ne pas prendre directement position.

On sait que les journaux de l'époque empruntent beaucoup les uns aux autres. Cela est vrai de tous les pays et la Chronik n'échappe pas à cette règle et il y a au moins deux publications dont elle ne fait pas mystère de s'inspirer: le Merkur de Wieland et Dohm ${ }^{21}$ qui fait alors autorité dans la presse allemande et un quotidien strasbourgeois, la Geschicbte der gegenwärtigen Zeit. Dans ce dernier cas, parler d'emprunt est un euphémisme, car c'est parfois de la reproduction quasi textuelle de colonnes entières qu'il s'agit. Le journal, créé le 1er octobre 1790 par deux Strasbourgeois luthériens, J. F. Simon et A. Meyer portait en exergue cette citation de Rousseau: «En tant que citoyen d'un Etat libre et membre de la puissance la plus grande, il est de mon devoir de m'informer des affaires publiques car j’ai le droit de dire mon mot à leur sujet, si peu d'influence qu'il puisse avoir.» Contrairement à E. Schneider qui rédige l'Argos en français, Meyer et Simon font choix d'écrire en allemand ${ }^{22}$.

La Geschichte der gegenwärtigen Zeit vivra jusqu'au 31 janvier 1793, devenant de plus en plus favorable à la cause révolutionnaire. C'est ainsi qu'après Varennes, elle suggère que l'on décapite le roi comme on le ferait s'il s'agissait d'un vulgaire porcher. Le ton ira ensuite se radicalisant. Mais, mort en septembre 1791 , Schubart ne se fera pas l'écho de cette radicalisation. Observons d'ailleurs que celle-ci n'empêche pas le journal strasbourgeois de demeurer opposé à toute idée d'égalité sociale. Aux privilèges, il entend substituer la hiérarchie des mérites et des talents. Tel est aussi le point de vue de Schubart, exprimé ici et là dans la Cbronik même si sur ce chapitre, il ne prend que rarement position ${ }^{23}$.

L'influence que le journal strasbourgeois exerce sur la pensée de Schubart n'est du reste pas exclusive, même s'il arrive qu'elle soit déterminante pour des durées limitées. Schubart y aura essentiellement trouvé des sources d'information, la traduction allemande des textes constitutionnels et leur commentaire, favorable certes, mais demeurant modéré dans le ton. Toutefois ce journal avait des liens étroits avec la Société des Amis de la Constitution qui souhaitait que Strasbourg servît d'exemple outre-Rhin. Elle désignait volontiers l'Allemagne comme la base de la contre-révolution, opposant

${ }^{21}$ Cf. Paul Hocks et Peter Schmidt, Literarische und politische Zeitschriften 1789-1805 (Stuttgart 1975) et Inge Stephan, Literarischer Jakobinismus in Deutschland 1789-1806 (Stuttgart 1976).

${ }^{22}$ Geschichte der gegenwärtigen Zeit. Sur la presse à Strasbourg à l'époque révolutionnaire, cf. E. Seinguerlet, Strasbourg pendant la Révolution (Paris 1881); Roland Marx, Recherches sur la vie de l'Alsace prérévolutionnaire et révolutionnaire (Strasbourg 1966); $R$. Oberlé, L'explosion révolutionnaire et ses conséquences (1789-1798), in: Georges Livet et François Rapp, Histoire de Strasbourg, des origines à nos jours, t.II (Strasbourg 1981) 549 sq.; de même Jacques Godechot, La Grande Nation, t.I (Paris 1956) 122 sq. qui souligne le rôle et la diffusion de la presse strasbourgeoise éditée en allemand.

${ }_{23}$ Cf. Gontbier-Louis Fink, Des privilèges nobiliaires aux privilèges bourgeois. Le débat sur l'égalité et son écho en Allemagne (1788-1792), in: Recherches germaniques 3 (1973) 30-101. Schubart souligne que les droits contenus dans la Déclaration ne sont rien sans la crainte de Dieu. Par ailleurs, il jugeait le droit naturel trop théorique. 
les "patriotes" aux émigrés qui trouvaient appui chez les princes au-delà du Rhin ${ }^{24}$. Mais c'est là un terrain où Schubart, à l'évidence, ne souhaite pas trop s'engager; malgré son peu de sympathie pour les émigrés, il préfère parler de ceux-ci en termes plus modérés.

En quels termes Schubart rend-il compte des événements de juillet 1789? Il faut attendre le 21 juillet pour que la Chronik les relate. Jusqu'alors Schubart semblait penser que, grâce à Necker, les plus grands périls avaient été surmontés et sa sympathie pour Louis XVI demeurait intacte. Le 17 juillet encore, il affirmait que le roi ne s'était pas coupé de la grande masse du peuple ${ }^{25}$. Mais voici que les choses se précipitent: dès lors, pour celui qui les juge au jour le jour, sans aucun recul, les impressions et partant les appréciations peuvent facilement être contradictoires. Ainsi Schubart annonce-t-il ce même jour que "la France entre dans une nouvelle période de son histoire, qu'elle ne tardera pas à secouer la poussière de l'esclavage et irradiera au soleil de la liberté. ${ }^{26}$ : avertissement, formule prémonitoire, manière voilée de faire état d'informations encore incomplètes? A moins qu'il ne s'agisse simplement d'une de ces affirmations grandiloquentes et volontiers prophétiques auxquelles Schubart, par tempérament, se laisse volontiers aller.

Le numéro suivant, daté du 21 juillet $^{27}$, est nettement plus explicite. Pour moitié, il est consacré aux événements parisiens et à la contagion qu'ils exercent sur des villes comme Orléans, Rouen ou Amiens. La Cbronik fait des émeutes parisiennes une description qui n'est pas très rassurante. L'heure est aux épées et aux couteaux. Le roi est menacé. Schubart lui conseille de ne pas se dérober. Qu'il se montre, face aux émeutiers, comme le père de son peuple. Sans doute a-t-il commis une grave erreur en renvoyant Necker. Mais le déchaînement de violence a de quoi effrayer. C'est le spectre de la guerre civile, avec toutes ses conséquences funestes, que voit se profiler Schubart. Et trois jours plus tard, il résume ainsi la situation: “Le meutre et le sang règnent à $\mathrm{Pa}$ ris.* Renforcée par d'autres émeutiers venus des provinces, la foule déchaînée a libéré les prisonniers de la Bastille.

Mais si l'on tient compte des délais alors nécessaires à la transmission des informations, il n'est pas surprenant qu'il faille attendre le 28 juillet ${ }^{28}$ pour que le journaliste prenne quelque distance par rapport à l'événement. Schubart en pressent la grandeur tragique qui serait digne d'inspirer la plume d'un Shakespeare ou d'un Schiller. Cependant il ne doute pas un instant que les bourgeois de Paris continuent d'être fidèles à leur roi. Par leur geste, ils ont montré que les Français souvent présentés, y compris par Schubart, comme des petits maîtres efféminés méritaient le cas échéant une autre réputation.

\footnotetext{
24 Schubart les tient pour anachroniques. Ils incarnent le prototype du Français superficiel et dominateur qu'il a naguère combattu. La Chronik voit d'autre part en eux une charge pour les Allemands, cf. Chronik, 6 septembre 1791, 589.

${ }^{25}$ Chronik, 17 juillet $1789,471$.

${ }^{26}$ Ibid. \&Frankreich beginnt jetzt eine neue Periode, und man wird sehen, wie es sich heben, den Staub der Sklaverei abschütteln und im Sommerschimmer der Freiheit ausstrahlen wird.•

${ }^{27}$ Chronik, 21 juillet $1789,475$.

${ }^{28}$ Chronik, 28 juillet $1789,489$.
} 
Autre enseignement à tirer de cette secousse qui, pour le moment, ne concerne que la France. Il s'adresse aux aristocrates allemands. Le tocsin de l'insurrection, pense Schubart, pourrait bien un jour sonner pour eux ${ }^{29}$.

A Strasbourg, dans un premier temps, le baron Klinglin a pu éviter le pire. Mais il n'a pas tardé à être débordé par l'émeute à laquelle se sont mêlés vagabonds et voleurs. Incendies, pillages, pendaisons, saccages de toute sorte marquent ces événements. La *flamme infernale» de l'insurrection gagne toute l'Alsace et même la Franche-Comté. Schubart ne minimise pas la gravité de ces événements. Il ne les idéalise pas davantage ${ }^{30}$. Il y a du reste un motif d'inquiétude plus précis: comment un sujet wurtembergeois pourrait-il se contenter d'observer calmement ce qui se passe dans ces deux provinces quand on sait que Montbéliard et Riquewihr sont possessions du duc de Wurtemberg? Le danger ne menace pas seulement des villes étrangères.

Il est du reste général. Schubart redoute de voir les émeutes se généraliser, de voir l'esprit de sédition gagner tous les esprits. La *colère des peuples* dont la Chronik du 31 juillet parle longuement revêt un double aspect ${ }^{31}$.

Sans doute a-t-elle des motifs bien réels: le mécontentement matériel et politique. Mais d'autre part elle relève de ce démon qu'est précisément l'esprit de sédition («Geist des Aufruhrs»): Schubart l'a toujours dénoncé. Et, de plus, ce phénomène risque de ne pas connaître de frontières. Non que Schubart se rallie à l'idée d'une conjuration internationale qui, par le truchement, ici des sociétés de pensée, ailleurs des loges maçonniques, aurait tissé sa trame à travers l'Europe entière. Il tient seulement qu'il s'agit d'une contagion qui, telle une traînée de poudre, ira enflammer les poudrières situées loin de son premier foyer d'explosion; cela d'autant plus que les motifs de mécontentement sont bien réels et perceptibles jusque dans les villes d'Europe où l'aristocratie abuse de son pouvoir.

Le mouvement dont les Français donnent l'exemple va-t-il être le signal d'un réveil des nations? Annonce-t-il le jugement dernier de l'histoire? Schubart ne sait s'il faut le souhaiter ou le craindre. Il emploie le terme, hégélien déjà, de «Weltgericht. On a cependant l'impression que chez lui l'angoisse prédomine devant ce raz-de-marée qui menace d'emporter toutes les formes de discipline et d'autorité. Ainsi, mu par la crainte, Schubart émet-il une appréciation franchement négative devant ce qu'il appelle la *flamme infernale*, "die höllische Flamme ${ }^{32}$.

L'insurrection, écrit-il, - car ce n'est pas encore de révolution qu'il s'agit - est un sous-produit des Lumières mal comprises, le résultat d'une hybris qui fait que l'homme prétend pouvoir se passer de Dieu et de l'autorité. La vraie cause des événements qui dévastent la France est peut-être là: c'est l'irreligion, le goût du blasphème, l'immoralité, une admiration béate, systématique et sommaire de la Constitution britannique, naguère tant prisée dans les colonnes de la Cbronik, et de l'exemple américain. Si Schubart ici établit une continuité entre les deux *révolutions», celle d'Améri-

29 Ibid., 493. .Die Sturmglocke wird seiner Zeit auch ihnen zum Schrecken hallen.

30 Ibid., 495

${ }^{31}$ Chronik, 31 juillet $1789,497-500$.

32 Chronik, 28 juillet 1789, 495. «Im ganzen Elsass prasselt die höllische Flamme. Auch in der Franche Comté stürmt's durcheinander.» 
que et celle de France, cette continuité est de nature toute négative et funeste. Ces tendances, déjà pernicieuses en soi, sont encore amplifiées par l'ignorance de la foule et le demi-savoir des sophistes, l'orgueil des orateurs et la crédulité du public. Tels sont, diagnostiqués par Schubart, les symptômes du mal qui n'épargnait en France aucune classe de la société et que la Chronik, au dernier jour du mois de juillet 1789 , désigne comme responsable de la secousse qui fait trembler le pays tout entier ${ }^{33}$.

Il arrive que, pour faire le point, Schubart insère dans la Chronik de courtes pièces en vers. Il l'a déjà fait en d'autres circonstances et c'est pour lui l'occasion de prendre un peu de ce recul qui lui fait défaut dans la relation immédiate des événements.

Ainsi, au début du mois d'août 1789 , la Chronik publie successivement deux poèmes consacrés au même sujet: la liberté. Le premier ne compte que huit vers. C'est un avertissement. Le peuple s'est fait illusion. Il ne sait pas faire usage de la liberté. Sous la forme qu'elle est en train de revêtir, la liberté est maudite par Dieu ${ }^{34}$.

A quelques jours de là, un plus long poème, onze strophes de cinq vers chacune, sous le titre Signe de ce temps ${ }^{35}$, exprime les mêmes préoccupations. C'est également une mise en garde face aux déchaînements sanglants dont s'accompagne parfois la liberté. Celle-ci est chose ambiguë: venue du ciel, elle est la sublime compagne des âmes sages et pieuses. Mais, entre les mains des peuples en furie, elle devient synonyme de vengeance et de dévastation. La sédition, dont la trompette résonne présentement à travers le monde, est qualifiée ici de splus noir des démons qui peuplent l'enfer».

Et pourtant! Lors même qu'il écrit ces lignes qui sonnent comme le désaveu de tout mouvement insurrectionnel, Schubart ne peut s'empêcher de saluer les acteurs de ces grandes journées. Comment ne pas être impressionné par cette foule où se mêlent fraternellement soldats, moines et bourgeois? La cassure ne porte-t-elle pas déjà en elle la promesse de la réconciliation ${ }^{36}$ ? Souvent, Schubart caresse ce rêve. Souvent aussi le spectacle de la réalité l'en détourne. Il est à craindre que les Français, par leurs excès, voient leur échapper cette liberté à peine conquise. D'ores et déjà des actes sanglants compromettent et déshonorent leur cause. On assiste à des scènes odieuses, comme l'exécution de Foulon et de son gendre Bertier. Dieu ne peut que détourner ses yeux de semblables crimes. Indigné, Schubart conclut son article par quelques vers où il met le peuple en garde contre le mauvais usage qu'il fait de la liberté. «Un peuple écla-

33 Ibid., 498. Plus de trois pages constituent un article intitulé *Der Völker Zorn* où Schubart écrit: Irreligion in allen Ständen, und bei den meisten herausvernünftelte Gottesleugnung; Unsittlichkeit; Anstaunen der englischen Konstitution ... der Aufenthalt vieler Offiziers in den amerikanischen Freistaaten ... etc. ... etc. ...“

${ }^{34}$ Chronik, août 1789,508 . Freiheit ... Wie wenig weiß ein Volk die Freiheit zu gebrauchen."

35 Chronik, août 1789, 508. Pour l'analyse de ces deux poèmes, cf. Gontbier-Louis Fink, La littérature allemande face à la Révolution française, in: Deutschland und die Französische Revolution, hrsg. von Jürgen Voss (München 1983) 249-301, notamment 265-267. Les contraintes de la tradition poétique, dont Schubart ne pouvait se défaire, font que c'est dans la prose de la Chronik qu'il s'exprimait avec le plus de netteté.

${ }_{36}$ Chronik, 4 août $1789,507$. 
boussé de sang est-il encore digne d'être libre?» „Non, répond Schubart: un tel peuple se précipite dans un esclavage pire encore que celui dont il a cru s'affranchir. ${ }^{37}$

Les rapports de ses correspondants strasbourgeois confirment les réflexions de Schubart sur les excès commis par la foule. L'hôtel de ville de Strasbourg a été pillé. Les bourgeois n'ont eu aucune part à ces exactions qui sont le fait de la *canaille* (das Gesindel») que Schubart oppose aux gens de bien («die Gutgesinnten») ${ }^{38}$. Bien que tout ce passage soit donné comme le récit d'un correspondant strasbourgeois, on peut admettre que Schubart fait siens les commentaires qui l'accompagnent. On peut même se demander si le correspondant en question n'est pas une fiction ...

Mais Schubart ne s'intéresse pas seulement aux événements qui ont pour théâtre la rue. L'aspect constitutionnel retient tout autant son attention. Il publie la liste des résolutions du 4 août. Il faut, dit-il en commentant ces dispositions, distinguer l'essentiel de l'accessoire. Or l'essentiel, c'est ce qu'il appelle «l'esprit élevé de la liberté gauloise*. Certes, de déplorables «bavures» accompagnent ces bouleversements ${ }^{39}$. Mais des douleurs de l'accouchement, pense Schubart, naîtra un «enfant gigantesque». Schubart raisonne et s'exprime ici un peu comme le fera plus tard Georg Forster qui, dans un passage dont les métaphores frapperont Hegel, évoquera la vertu fécondante des orages dévastateurs et le ciel serein qui succède à leur déchaînement ${ }^{40}$.

Pour l'heure, cet «esprit de la liberté lui paraît s'incarner dans la Constitution: la traduction qu'en donne la Chronik est d'ailleurs celle qu'il a pu trouver dans les journaux strasbourgeois qui le tiennent informé, la Geschicbte der gegenwärtigen Zeit notamment. Mais, dans le numéro suivant, c'est-à-dire le 1er septembre, il dénonce les honteuses exactions dont Paris est le théâtre. Le fruit jeune et tendre de la liberté pourra-t-il s'épanouir dans un tel climat, se demande-t-il? Elargissant le débat, il écrit cette phrase que n'eût pas désavouée Wieland: «Il semble que le peuple ne puisse pas supporter le bonheur de la liberté. ${ }^{41}$

Prenant un peu de recul, Schubart consacre la quasi-totalité du numéro LXXIII à un dialogue qui met en présence deux personnages, pour la circonstance nommés Gottholdt et Warnfried ${ }^{42}$. Cet échange d'opinions, plus complémentaires qu'antagonistes, se situe le 11 septembre 1789. Le dialogue en question s'intitule .Sédition et Révoltew. C'est pour Schubart l'occasion de faire l'apologie du prince avisé qui, par des réformes faites à temps, évite à son peuple d'être atteint par la contagion d'un exemple

${ }^{37}$ Chronik, ibid., 508. «Ein Volk, bespritzt von Blut, verdient nicht frei zu sein. In härtere Sklaverei stürzt es sich allein*.

38 Ibid., 505 sq., Schubart emploie ici le mot «Gesindel» qu'il oppose aux «Gutgesinnte. Il juge donc en fonction de catégories morales plus que selon des critères sociaux.

39 Chronik, 11 août 1789,521 et 526 .

40 Ibid. .Wenn ein Meer austritt, so geusst es verpestenden Schlamm ans Ufer.* Des métaphores analogues, mais employées dans une acception positive, se trouvent chez Forster. Hegel avait lu attentivement les Ansichten vom Niederrhein (cf. Jacques D'Hondt, De Hegel à Marx (Paris 1972) 74 sq.) et cite la phrase suivante de Forster: *Wir wünschen unseren Pflanzungen Gewitterregen, wenn gleich zuweilen durch den Blitz ein Dorf zum Raub der Flammen wird.. Le 17 septembre 1790, la Chronik écrira: ،Getrost und unverzagt, Franke, erst nach dieser Sichtung beginnt die Periode der Glückseligkeit.»

${ }_{41}$ Chronik, 22 septembre 1789,641 et 643.

42 Chronik, 11 septembre 1789,613 sq. 
qui se discrédite de lui-même. L'Allemagne, terre de concorde où règnent ces vertus quotidiennes que les moralische Wochenschriften avaient préconisées tout au long du XVIIIème siècle, devrait rester à l'abri du danger. Mais là encore, le débat s'élargit. Ce qu'il faut, c'est d'abord acquérir la liberté intérieure. Vouloir aller trop vite est une dangereuse chimère. Au demeurant, la vraie liberté n'est pas de ce monde; elle ne prospère vraiment qu'au royaume des cieux. Mais l'essentiel est que l'avertissement soit entendu, que les princes allemands tirent la leçon du mal qui frappe leur voisin pour ainsi en prévenir l'extension.

Warnfried se dit entièrement d'accord avec Schirach que Schubart évoque fréquemment en ces débuts de la Révolution Française. Cette question: comment prévenir le phénomène révolutionnaire? est bien celle qui préoccupe les intellectuels d'outreRhin $^{43}$, qu'ils soient, comme Schirach de tendance modérée, ou qu'ils passent, comme Rebmann ou Knigge pour professer des opinions plus hardies. On ne sera donc pas surpris de voir Schubart se rallier ici à un point de vue qui fait presque l'unanimité dans la pensée allemande à cette époque. Notons cependant que, à l'instar de Schirach, il aborde ce problème dès les premières semaines de la Révolution, alors que, pour beaucoup, il ne s'imposera que deux ou trois années plus tard.

D'ailleurs Schubart ne nie pas que de ce chaos et de cette fermentation naissent les initiatives les plus généreuses. On peut ainsi observer une véritable métamorphose du caractère national français ${ }^{44}$. Les femmes françaises, pourtant frivoles et volages de nature, accèdent à la dignité et à l'esprit de sacrifice dignes de l'Antiquité. Comme nombre d'Allemands, Schubart est impressionné par cette résurgence du patriotisme à l'antique. Dès qu'il croit percevoir les chances d'une «monarchie modérée, Schubart espère de nouveau. Quelque chose de positif émerge malgré les facteurs de déception. Cet espoir s'incarne dans la Constitution, l'esprit de liberté qui inspire toute chose, la liberté de la presse, cause chère entre toutes au journaliste Schubart (mais qu'on prenne garde à l'usage qui en sera fait!) et la tolérance en matière religieuse que réclame si éloquemment Rabaut-Saint-Etienne.

Quant aux pays allemands, on peut espérer qu'ils demeureront à l'abri. Encore une fois, Schubart est ici bien proche de Wieland et de tous ceux qui constituent ce qu'on appelle habituellement la "réaction idéaliste ${ }^{45}$. La Cbronik dément avec plaisir l'information venue de Suisse et faisant état de soulèvements survenus dans ce pays. Ainsi peut-on lire, toujours à cette date du 1er septembre 1789: ‘Voilà qui réconforte mon âme et réjouit mon cœur: de voir qu'il existe encore des Etats où l'Autorité et ceux qui y sont soumis, chacun en connaissance du caractère sacré de ses devoirs, demeurent attachés l'un à l'autre par des liens de confiance et d'amour, comme le sont des parents

${ }^{43}$ Cf. par exemple le baron Knigge, Josephs von Wurmbrand politisches Glaubensbekenntnis, mit Hinsicht auf die französische Revolution und deren Folgen (Bremen 1792, éd. G. Steiner, Frankfurt 1968) le 8ème chapitre, intitulé: .Wie allen gewaltsamen Revolutionen vorgebeugt werden könne, 98 sq.

44 Chronik, 18 septembre 1789, 632. «Unter dieser anscheinenden Anarchie geschehen Kopfund Herztaten, die ewiger Aufbewahrung wert sind.

45 Cf. Droz, 297 sq. La réaction humaniste. 
et des enfants chrétiens. ${ }^{46}$ La vision de la Suisse, jusqu'alors pays des âmes où souffle l'air pur de la liberté, s'enrichit ici d'une vision patriarcale: la liberté devient facteur d'harmonie et non pas ferment de discorde. L'image que Schubart donne de ce pays en fait un modèle de régime équilibré qui le met à l'abri des secousses que l'on observe à Paris où s'installe la misère, où prolifère une armée de fainéants, de parasites et de pillards, cependant que l'esprit de revendication tend à gagner toutes les corporations.

Maladies infantiles de la Révolution? Sans doute, et qui ne doivent pas faire condamner absolument celle-ci ni les principes qui la guident même si elles risquent de rendre périlleux chaque pas accompli sur le chemin de la liberté et du bonheur du peuple. Que la preuve de cette incapacité à assumer la liberté tant revendiquée soit fournie par le peuple français n'est pas fait pour étonner Schubart. Son inconstance et ses autres défauts ne pouvaient pas disparaître en un jour. C'est déjà l'idée, qui sera amplement développée à l'époque napoléonienne, selon laquelle le caractère français galvaude inévitablement les causes les plus sacrées, autrement dit que les Français ne sont pas vraiment dignes des principes qu'ils prétendent incarner. Son corollaire qui veut que les Allemands, qui possèdent précisément les vertus qui font défaut aux Français, soient particulièrement aptes à incarner ces valeurs suprêmes et même soient désignés pour le faire, ne figure pas encore chez Schubart. Il n'apparaîtra que plus tard, à l'époque des guerres napoléoniennes.

Schubart, pour l'heure, se contente de dire que les Allemands auraient bien tort de vouloir imiter ce que les Français ont l'audace d'appeler «liberté et qui n'est souvent que "folie furieuse*. Le cheval emballé a déjà jeté bas son cavalier. Bientôt, il se rompra les membres et le cou ${ }^{47}$.

La France révolutionnaire offre ainsi à vrai dire une double image: celle du désordre, de l'anarchie sur fond d'illusions qui mènent tout droit à la banqueroute. Et celle de la réconciliation nationale. Paris corrobore la première impression, Strasbourg confirme la seconde puisque, dans cette ville, la réconciliation met même fin aux querelles où s'opposaient catholiques et protestants. Laquelle de ces deux images est la bonne? C'est selon les informations que Schubart reçoit.

Mais précisément la Fête de la Fédération va être pour Schubart l'occasion de concrétiser ce vœu. Cette fête a fortement frappé les Allemands, qu'ils en aient été les témoins - comme Schubart lui-même qui fit le voyage de Strasbourg quelques semaines avant sa mort - ou qu'ils en aient seulement été informés par leurs journaux ou par des récits de voyageurs. Citons par exemple G. A. von Halem ${ }^{48}$, ami et admirateur de Klopstock et de Wieland. Comme beaucoup de ses compatriotes, il souligne le symbole de fraternité nationale et d'unanimité que constitue cet événement. Il y perçoit ce qu’y éprouve Michelet qui écrit: “La France de 89 a senti la liberté celle de 90

${ }^{46}$ Chronik, ler septembre $1789,586: « \ldots$ in Vertrauen und Liebe aneinander hangen, wie christliche Eltern und Kinder..

47 Chronik, 4 septembre 1789,595 et 11 août, 525 sq. et 11 septembre 1789,615 .

48 G. A. von Harlem. On peut se rapporter à la traduction d'Artbur Chuquet, Paris en 1790. Voyage de Harlem (Paris 1896). Sur G. A. von Harlem, cf. Karsten Witte, Reise in die Revolution. G. A. von Harlem und Frankreich im Jahre 1790 (Stuttgart 1971) notamment 75, 77, 90 et 91. 
sent l'unité de la patrie»; Michelet parle encore de «cet immense élan de concorde au bout d'un siècle de discorde». A propos de la manière dont les Allemands ont ressenti cet événement, il ajoute: «L'Allemagne, devant ce miracle, fut profondément absorbée, entre le rêve et l'extase, devant ce spectacle de l'union qui gravite à l'unité.\$\$9

C'est bien ainsi que Schubart nous décrit cette journée: unité de tous les Français, réconciliation du roi et de la nation, donc fête nationale en tant qu'elle symbolise ce double phénomène. Son émotion est encore plus grande du fait que, dans un club parisien, on a bu ce jour-là à la santé de Wieland qui, dans le Merkur, a pris position en faveur de la Révolution, cependant qu'on y brûlait les numéros des Staatsanzeiger où Schlözer prenait la défense des aristocrates ${ }^{50}$.

Le premier écho de ces journées que nous livre la Cbronik ne concerne pas.Paris mais Strasbourg. Schubart compare cette fête à une parfaite symphonie, majestueusement exécutée et que ne dépare aucune fausse note («ohne Mißlaut" écrit Schubart). Harmonie, concorde, patriotisme vrai, c'est aussi ce que Pfeffel, le poète aveugle de Colmar, a ressenti en ce jour qui est celui de la résurrection de la patrie: clivages sociaux et divisions provinciales disparaissent spontanément ${ }^{51}$.

Schubart souscrit à la double partition de cette symphonie: d'une part, l'idylle que constitue la réconciliation du roi et de la nation, le rêve, devenu réalité, d'un despotisme éclairé et populaire; d'autre part, le sentiment tout puissant d'appartenir à la nation française, donc à une patrie qui l'emporte sur tous les particularismes provinciaux. Pour un Allemand qui a rêvé de l'impossible unité des Allemagnes, quel exemple! Sur ce chemin les Français montrent la voie où Schubart aimerait voir ses compatriotes s'engager. Seuls existent à présent le peuple de France et les Français, qu'ils soient Bretons, Normands ou Provençaux. Comme Schubart aurait aimé qu'il en fût de même des Wurtembergeois, des Saxons, des Bavarois et de tous les Allemands dispersés dans l'Allemagne émiettée. Jamais poète, ajoute Schubart, n'aurait osé rêver une telle fraternité étendue à tout un peuple 52 .

Tous ont contribué à la préparer, quelles que fussent leurs origines, sociales ou régionales. N'a-t-on pas vu le roi pousser lui-même la brouette? Fraternelle chaleur qui embrasait aussi bien les prêtres et les moines, les enfants et les femmes, les soldats, les savants, les artistes. Le roi, rallié à la Constitution à laquelle il prête serment, promet d'exécuter les lois. N'est-ce pas la meilleure garantie que l'hydre de l'anarchie n'est plus à craindre? Cet enthousiasme s'étend même aux étrangers. A. Cloots en est l'exemple le plus éloquent. Lui, un Prussien, au cœur vaste et à l'esprit élevé, a jeté aux orties son titre de baron - et le voilà qui pousse la brouette! Cloots s'était proclamé

49 Jules Michelet, Histoire de la Révolution Française (chapitre XII: 14 juillet 1790). Nous citons d'après l'édition de C. Mettra, I (Paris 1979), 332 sq.

so Chronik, 22 juin $1790,431$.

${ }^{31}$ Chronik, 22 juin 1790, 431: «Das ganze große Fest glich einer vollständigen Symphonie, ohne Mißlaut, herrlich gesetzt, und majestātisch ausgeführt.* Cf. Gonthier-Louis Fink, Pfeffel et la Révolution Française, in: Recherches germaniques 17 (1987) 121 sq.

52 Chronik, 25 juin 1790. AAlle Rivalităt ist erloschen; es ist jetzt nur ein Volk ... 
cosmopolite parisien et clévois de naissance tout à la fois. Il s'était exclamé: „Voilà la Gaule, vive l'humanité.»

Du Ca ira, Schubart retient deux vers:

A ça ira, ça ira

Nos chers frères des Provinces

qu'il préfère à ceux de contenu plus égalitaire ${ }^{53}$.

Ici Schubart risque un rapprochement, qu'il ne développera pas par la suite, entre la Révolution Française et la guerre d'Indépendance des Etats-Unis. La Fayette est le symbole de cette double liberté. Schubart va même jusqu'à écrire que la Révolution Française, œuvre de raison, se distingue de la liberté conquise par les Américains en ceci qu'elle ne fait pas couler des flots de sang ... ${ }^{54}$.

Pour nous replacer dans la perspective qui était celle de Schubart et de ses lecteurs, il nous faut ignorer ce qui arrivera après l'événement qu'il rapporte et commente, nous arrêter au jour où il écrit. Il faut donc renoncer à la position de l'historien qui dispose du recul nécessaire et confirme un tri que le cours des choses a déjà opéré avant lui. Cela est naturellement encore plus vrai lorsqu'il s'agit d'une actualité aussi brûlante que celle-ci et dont le déroulement précipité est totalement imprévisible.

La vision que transmet la Chronik est donc à la fois insuffisante mais précieuse. Elle manque, et pour cause, de la profondeur de champ dont dispose l'historien, mais en revanche, elle nous renseigne sur ce que l'histoire a parfois oublié. La portée d'un événement ne coïncide pas toujours avec ce que ceux qui l'ont vécu avaient primitivement pensé, avaient espéré ou avaient redouté.

La fête de la Fédération en fournit un bon exemple. L'historien sait ce que sera le 14 juillet 1791. Il pourra être tenté de dire que l'année 1790 qui culmine avec la Fête du Champ de Mars a été l'année heureuse de la Révolution ${ }^{55}$. Cela implique que celles qui l'ont suivie ne méritent pas ce qualificatif. D'autre part, la connaissance de l'évolution ultérieure permet de souligner ce que cette unanimité masquait de conflits. Mais le journaliste fournit un témoignage infiniment précieux pour celui qui veut ignorer ce qui viendra plus tard.

«Pour comprendre ce qu'a signifié cette journée, écrit F. Furet, ignorons volontairement ce qui va venir. Imitons ces bourgeois de l'Ancien Régime qui enregistraient dans leur journal les impressions du temps.. ${ }^{56}$ C'est de même un récit pour ainsi dire au jour le jour que nous livre Schubart. Tout au plus se permet-il quelques retours en arrière, assez rares et limités. Il tente alors de dresser un bilan, conscient qu'il est de devoir accompagner le rythme précipité des événements et d'ajouter des objections à un contexte déjà fort contradictoire en lui-même.

${ }^{53}$ Chronik, 23 juillet 1790, 503. La moitié de ce numéro s'intitule: „Das Fest der Freien.* Cf. déjà le 20 juillet $1790,495-498$.

54 Ibid. 504.

55 Cf. Fransois Furet, La Révolution, t. 1 (Paris 1965) 164. Jacques Solé dans La Révolution en questions (Paris 1988) 112 sq. font référence à Samuel Scott qui ironise sur al'année heureuse. que fut 1790. Jean Tulard, Histoire et dictionnaire de la Révolution française (Paris 1987) 65 sq. Schubart a bien vu que s'opérait là une nouvelle naissance de la France. Mais il n'analyse pas la fragilité de cette unanimité et préfère quelque temps poursuivre son rêve idyllique.

56 Furet, 164. 
L'année 1791, qui verra la disparition de Schubart, sera marquée par deux événements qui eurent en Allemagne une immense répercussion: l'entrevue de Pillnitz et la tentative de fuite de Louis XVI qui prit fin à Varennes. De l'un et de l'autre de ces événements, la Chronik a rendu compte et a essayé de tirer des conclusions allant au-delà de la seule relation des événements.

Dans l'entrevue de Pillnitz, Schubart voit avant tout un fait de nature à renforcer les liens du corps germanique. Aussi bien les deux événements ont-ils entre eux le lien que l'on sait. La chronologie nous commande de même de commencer par la fuite du roi. Depuis quelque temps, la Cbronik s'inquiétait des agissements des adversaires de la Révolution. Sans doute était-ce pure calomnie, disait Schubart, si l'on prétend que le roi est impliqué dans ce complot. «Il faut faire litière, ajoute-t-il, des affirmations selon lesquelles le roi conspirerait ainsi à la défaite de son propre peuple. ${ }^{57}$ Schubart, encore une fois, est partagé. Il se refuse à croire ceux qui prêtent au roi l'intention de fuir: les émigrés de Coblence se bercent assurément d'illusions. Mais la confusion, ce que Schubart appelle ici la «Schwärmerei», étendant au politique l'acceptation habituellement psychologique et littéraire de ce mot toujours négatif sous sa plume, règne dans les deux camps. La cruauté qui sévit dans les prisons ne vaut pas mieux que celle qui sévissait à la Bastille. L'Assemblé Nationale ne devrait être fréquentée que par des patriotes purs et enthousiastes. Hélas, les fanatiques y sont au moins aussi nombreux et la concorde espérée est loin de régner en France. Par ailleurs, Schubart partage sur le destin de la France le pessimisme d'Archenholtz dont il cite les articles parus dans Minerva ${ }^{58}$.

La fuite du roi elle-même est largement commentée le 1er juillet. Après avoir fait le récit des événements, Schubart conclut: « $\mathrm{Si}$ la tentative du roi avait réussi, la pire des guerres civiles se serait déchaînée sur le pays; les armées étrangères auraient déferlé, ralliant les mécontents. ${ }^{59}$ Aussi sait-il gré à Drouet d'avoir arrêté cette dangereuse expédition. Schubart tient pour acquise la collusion entre la famille royale et les émigrés, prêts à intervenir aux frontières. Sans accabler Louis XVI, Schubart se félicite que sa tentative ait échoué. L'avenir dira ce qu'il adviendra du roi. Beaucoup de choses dépendent de l'empereur Léopold.

Quelques jours plus tard, la Chronik relate l'entrée de Louis XVI dans Paris. Il est surtout frappé par l'accueil qui lui est fait. Schubart conclut en ces termes: «Qui n'accorderait pas sa pitié au roi, qui assurément n'est pas mauvais, mais seulement faible.» Et il se laisse aller à cette prophétie: cet événement préfigure le jour ou tous les rois, princes et seigneurs de la terre devront rendre des comptes ${ }^{60}$.

57 Chronik, 24 juin 1791.

${ }^{58}$ Cf. Jacques D'Hondt, Hegel secret (Paris 1968) chapitre premier. .Minerva* 7-43. Archenholtz, fondateur et directeur de cette revue, apparaît comme un modéré, proche des Feuillants et qui, plus tard, se montre très hostile aux Jacobins. On lui doit par ailleurs une Histoire de la Guerre de Sept Ans à laquelle il avait lui-même pris part comme officier dans l'armée prussienne.

59 Chronik, notamment 1er juillet 1791, 495. «Wäre dem König seine Entweichung gelungen, so würde sogleich der fürchterlichste Bürgerkrieg ausgebrochen sein.*

${ }^{60}$ Chronik, 5 juillet 1791, 437. Par ailleurs la Chronik publie la traduction intégrale de la lettre adressée par le Marquis de Bouillé à l'Assemblée Nationale. 
Mais qu'allaient être les conséquences de cet événement en Europe? Le 2 septembre, la Chronik annonce en ces termes l'entrevue de Pillnitz: „Au mois d'août, Léopold et Frédéric-Guillaume s'entretiennent de choses de la plus haute importance.» Une semaine plus tard, Schubart précise: «Bien que cet accord ressemble fort à l'atelier de Vulcain où se forgent les massues destinées à terrasser la Constitution Française, il est plus vraisemblable pour qui connaît les caractères des deux souverains que ceux-ci éviteront l'intervention violente. ${ }^{61}$

De Pillniz, Schubart retient essentiellement une image: celle d'une embrassade qui met fin à des siècles de discorde opposant la Prusse à l'Autriche et qui, ainsi, inaugure un nouveau système ${ }^{62}$. Le fait le plus significatif est que Schubart accentue cet aspect, certes indéniable, de l'entrevue de Pillnitz mais qu'il tend de ce fait à en minimiser la portée contre-révolutionnaire ${ }^{63}$. Louis XVI ayant accepté la Constitution, l'accord en question perd de son actualité, en tout cas de sa virulence.

Schubart ferme donc au moins en partie les yeux sur l'affrontement qui apparaît de plus en plus inévitable entre les princes et la Révolution. On observera que d'autres périodiques font alors preuve d'une plus grande lucidité. C'est le cas du Deutsches $M u$ seum qui écrit vers le même moment: «C'est une remarque incontestable et que chacun peut faire: ou bien, il faudra que les princes d'Europe vengent la honte subie par Louis XVI, ou bien il faudra qu'ils s'attendent à subir eux-mêmes un sort identique. Ou bien il faut extirper l'anarchie, ou bien celle-ci contaminera l'Europe entière et elle rendra tous les pays aussi malheureux que l'est la France. ${ }^{64}$ Approfondissant son analyse du rapport des forces en Europe, la même revue insiste sur le changement de perspective à ses yeux décisif que signifie la conclusion avec la Turquie de la paix de Sistowa, le 4 août 1791 : l'Autriche, qui a désormais les mains libres à l'Est, va pouvoir s'intéresser de beaucoup plus près à ce qui se passe à l'Ouest. La Chronik signale l'événement mais n'en tire pas de conclusions. Tout au plus évoque-t-elle l'hypothèse d'un changement de priorité désormais possible dans la politique extérieure de l'Autriche.

Schubart en reste donc, s'agissant de Pillnitz, à l'image d'une réconciliation austroprussienne. Certes, cet accord sonne le glas du Fürstenbund que la Chronik avait pourtant très vivement célébré et qui, rappelle Schubart, a été le grand espoir des patriotes.

${ }^{61}$ Chronik, 2 septembre 1791, 578.

${ }^{62}$ Chronik, 13 septembre 1791, 599. «Hat nach langer blutiger Zwietracht des Preußischen Genius den Oesterreichischen umarmt, und ein neues Staatssystem geschaffen.*

${ }^{63}$ Chronik, 27 septembre 1791, 634. De même, c'est sous l'angle de l'unanimité nationale allemande que Schubart envisage ce qu'il appelle la egrande affaire alsacienne*. La riposte du Reichstag aux décisions françaises concernant les «princes possessionnés d'Alsace. lui est prétexte á célébrer le rassemblement de tous les Allemands (Chronik, 5 août 1791, 512) dès lors que l'Empire est menacé dans son intégrité territoriale, et à souligner l'appartenance de l'Alsace au corps germanique (Chronik, 23 septembre 1791). La position de la Chronik, même sur ce dernier point, n'est d'ailleurs pas inébranlable. Schubart, en tout cas, tient à se démarquer des esanguinaires» qui prèchent à cette occasion la croisade antifrançaise (Chronik, 30 novembre 1790, 818).

${ }_{64}$ Neues Deutsches Museum 1791, 849, cité par Waldemar Wenck, Deutschland vor hundert Jahren (Leipzig 1890) Bd. II, 197. 
Pillnitz, Schubart doit bien l'admettre, c'est un peu le désaveu de l'œuvre de Frédéric ${ }^{65}$. Mais ses regrets semblent comme effacés. Il tient pour éminemment positif tout ce qui renforce les liens entre les différents éléments du corps germanique. L'unité du monde allemand passe à ses yeux avant la vénération qu'il peut avoir pour ses illustres représentants, lors même que ceux-ci ont, en leur temps, contribué à édifier cette unité. Certes, les petits Etats allemands dont la Chronik a souvent dit de quelle riche diversité ils étaient porteurs, risquent d'y être sacrifiés. Les princes de ces Etats, dit Schubart, ne voient pas l'abîme dans lequel ils se précipitent. Ils portent du bois au bûcher qui va les anéantir. Un correspondant, réel ou fictif de Schubart, se pose au moins la question.

Dans les derniers mois de la vie de Schubart, la Chronik se contente d'être un journal d'information qui évite de prendre parti, sans doute à fois par prudence personnelle de son éditeur et aussi parce que celui-ci se sent débordé par la précipitation souvent contradictoire du cours des choses. Comment, dans ces conditions, s'aventurer à prédire? Ce serait courir le risque de devoir se démentir quelques jours plus tard.

Dans la Chronik de l'été 1791 , on peut trouver des informations, beaucoup d'interrogations et d'hésitations. Mais, si l'on excepte une approbation de principe envers tout ce qui semble de nature à fortifier l'Allemagne, de plus en plus perçue à travers la personnalité de Léopold, on ne trouvera guère de prises de position politiques. Il ne faut pas y chercher l'écho du grand débat qui s'amorce ailleurs: par exemple, dans le Neues Deutsches Museum ou dans le Wiener Zeitschrift qui accentuent leur pression en faveur du soutien accordé à Louis XVI et d'une intervention armée destinée à contenir, voire à réduire la Révolution; mais aussi, dans le camp opposé, chez un homme comme Forster qui escompte des préoccupations que la Prusse et l'Autriche auront à l'Est un effet modérateur quant à leur volonté d'intervenir en France et n'hésite pas à dresser au même moment un bilan positif de la Révolution.

Cette prudence dans le jugement, cette hésitation à s'engager chez Schubart que nous avons connu si impétueux, rejoignent une attitude très comparable que l'on peut observer chez Wieland qu'un critique récent qualifie de «bourgeois sceptique ${ }^{66}$. C'est d'ailleurs à cet écrivain que se réfère volontiers Schubart dans l'un des tout derniers numéros de la Chronik qu'il rédigea avant sa mort ${ }^{67}$. Ce qu'espère Wieland, qui ne souhaite en rien l'extension à l'Allemagne d'une Révolution qui remettrait en cause trop de choses auxquelles il est attaché, c'est que précisément les princes allemands veillent à préserver cet équilibre fragile plutôt que d'aller guerroyer contre un grand peuple qui a brisé ses chaînes avec l'énorme énergie que donne le désespoir. Le spectateur sceptique et l'observateur passionné se rejoignent ici. C'est une leçon de sagesse assez conservatrice que Schubart croit pouvoir dégager des Nouveaux Dialogues des Dieux que Wieland vient justement de publier à Leipzig. «Ils contiennent, écrit la

65 Chronik, 13 septembre 1791, 601 et 27 septembre 1791, 632. Schubart fait parler un Franconien qui regrette l'affaiblissement de la Prusse.

${ }^{66}$ Chronik, 4 octobre 1791, 650 sq.

${ }^{67}$ B. Weyergraf, Der skeptische Bürger. Wielands Schriften zur Französischen Revolution (Stuttgart 1972); cf. également Gonthier-Louis Fink, Wieland et la Révolution Française, in: Revue d'Allemagne (juillet 1973) 497-522. 
Chronik, les vérités les plus splendides concernant les gouvernements et les Constitutions." A l'appui, Schubart cite un passage de ces Dialogues où l'on peut lire que toute entreprise menée contre la liberté constitue une prétention indue à l'égard du plus inaliénable des droits naturels que possède un être raisonnable. Le passage s'achève sur cette phrase: "Plus les hommes discernent clairement le pour et le contre de toute chose, moins ils seront enclins à échanger contre des inconnues et des incertitudes leur situation présente, pour peu que celle-ci ne soit pas totalement insupportable. ${ }^{68}$

Cela est surtout vrai des Allemands dont la Cbronik remarquait le 26 juillet 1791 , s'adressant à ceux de ses lecteurs qui croyaient remarquer les étincelles annonciatrices d'un proche incendie: «L'Allemand est solidement calé dans la chaise de son grandpère; il est enclin, plus que les autres nations, à se satisfaire de son sort, à la soumission, au travail. Il réfléchit et pense: en ce monde on ne peut pas encore trouver la vraie liberté. C'est seulement dans l'au-delà qu'il nous sera donné de contempler la loi parfaite de la liberté., ${ }^{69}$

Sans doute, ceux que la Chronik depuis un peu plus de deux ans, nomme les Nouveaux Francs, à la veille de se donner une nouvelle Constitution, peuvent-ils céder à une certaine euphorie. Schubart croit percevoir chez eux l'annonce d'une véritable réconciliation nationale. L'avant-dernier numéro de la Chronik composé avant la mort de Schubart est presque entièrement consacré à la France. Il y célèbre successivement Louis XVI et Rousseau. Il met en garde contre les Emigrés coupables, selon lui, de menées contre-révolutionnaires. L'ancien droit - das alte Recht ${ }^{70}$ - et le nouveau ne sont, dit Schubart, nullement incompatibles. Dans les pays allemands, et d'abord en Wurtemberg, l'ancien droit protégeait du despotisme. Le nouveau droit est né de l'insurrection contre le même adversaire ${ }^{71}$.

Dans les dernières semaines de sa vie, Schubart semble se laisser porter par un élan révolutionnaire plus vif qu'auparavant. Certes des ombres subsistent au tableau, perspectives de guerre civile notamment, que redoute tant $W$ ieland. Chez Schubart, alternent moments d'optimisme et accès de pessimisme, visions de discorde et visions de réconciliation.

Mais même s'il lui arrive de déplorer que ses compatriotes mettent si peu d'enthousiasme à entendre le message venu de France, même s'il ne rejoint pas le camp des admirateurs devenus détracteurs - pour la bonne raison que, mort en octobre 1791, il n'a pas connu la suite des événements - Schubart a toujours pensé que le mieux était de faire l'économie de la Révolution, surtout lorsque l'on est Allemand et sujet du duc de Wurtemberg. Si la flamme de la Révolution doit se propager, mieux vaut qu'elle le fasse dans une autre direction!

68 Ibid., 651.

${ }^{69}$ Chronik, 26 juillet 1791,489 sq. Schubart consacre trois pages entières à décrire la paix enviable qui règne en Allemagne; il conclut ainsi: «Ich mußte diese kurze, aber wahre Darstellung denjenigen entgegenstellen, die jetzt in Deutschland glimmende Funken eines wahren Brandes bemerken wollen. Der Deutsche sitzt fest und breit in seinem Großvaterstuhle und ist zur Genügsamkeit, zur Unterwerfung, zur Arbeit geneigter, als andere Nationen. Er ist verständig und denkt: drüben erst wird es uns glücken, hineinzuschauen in das vollkommene Gesetz der Freiheit.•

70 Cf. Erwin Hölzle, Das alte Recht und die Revolution (Oldenburg 1931) 60 sq.

71 Chronik, 7 et 11 octobre 1791, 656 sq. et 663 sq. 


\section{Zusammenfassung des Referates von Jean Clédière}

Schubart war bereits seit zwei Jahren aus dem Gefängnis von Hohenasperg entlassen, als am 21. Juli 1789 die Vaterländische Cbronik, die er künftig in der Druckerei der Karlsschule herausgab, diese UUberlegung an ihren Anfang stellte:

In Frankreich geschehen jetzt Dinge von solcher Wichtigkeit, daß man fast darüber die Donner des Türkischen und Nordischen Krieges überhört.

(Vaterländische Chronik, LVIII, S. 473, Dienstag, 21. 7. 1789)

Seit dieser Zeit erhielten die Leser der Zeitschrift regelmäßig Informationen über die Ereignisse in Frankreich, insbesondere aus Paris und Straßburg, wo Schubart über Korrespondenten verfügte, deren Identität er jedoch niemals offenbarte.

Die geographische Nähe wie auch eine gewisse freiheitliche Tradition auf dem Gebiet des politischen Denkens ließen Württemberg damals besonders sensibel auf diese Ereignisse reagieren. Schubart erhielt seine Informationen hauptsächlich aus Straßburg und ging so weit, sich fast wörtlich von gewissen Straßburger Zeitschriften - wie der Geschichte der gegenwärtigen Zeit - inspirieren zu lassen. Er reagierte mit Sensibilität und Impulsivität, was zeigt, daß ihn die zehn Jahre der Gefangenschaft nicht gebrochen hatten, selbst wenn er wegen Karl Eugen verstāndlicherweise gewisse Rücksichten nahm.

Die Vaterländische Chronik erschien zweimal wöchentlich und bemühte sich, den aktuellen Stand der Ereignisse darzustellen. Es darf nicht verwundern, daß in ihr oft stark unterschiedliche Einschätzungen zu finden sind, mitunter sogar Widersprüche. Die Ereignisse entwickelten sich sehr schnell. Ihre Beurteilung und mehr noch der Versuch einer Prognose waren sehr schwierig. Schubart bedauerte, daß ihm die $Z_{u}-$ rückgezogenheit und Zufriedenheit eines Historikers versagt geblieben seien. Außerdem erklärt auch die durch die Überwachung Karl Eugens bedingte ambivalente und kluge Haltung, von der die Tagebücher seiner Rayssen zeugen, daß Schubart zögerte, sich zu deutlich zu engagieren. Er praktizierte von nun an eine Art Selbstzensur, die die Voraussetzung für die Neuausgabe seiner Zeitschrift gewesen ist.

Zweifellos war Schubart von vornherein entschlossen, die begeisterte Stimmung zu unterstützen, die sich vieler junger Intellektueller Württembergs bemächtigte. Diese waren in Karlsschule und Tübinger Stift erzogen worden. Treu blieb von ihnen G. Stäudlin, der versuchte, die Chronik nach dem Tod ihres Gründers fortzuführen.

Um die universelle Bedeutung des Ereignisses anzudeuten, zögerte Schubart nicht, seine Zeitschrift ganz kurz Chronik zu nennen. Er, der die Franzosen so sehr verächtlich gemacht hatte, bewunderte sie jetzt und war sogar bereit, bei ihnen etwas wie einen deutschen Geist zu erkennen. Dennoch befürchtete er die endgültige Französisierung von Straßburg, das in seinen Augen ein Brennpunkt deutscher Kultur blieb. Auch traute er den Franzosen nicht zu, mit der Freiheit, die sie für sich in Anspruch nahmen, wirklich leben zu können. Schubart nahm also die Akzente der antifranzösischen Polemik wieder auf, mit der er an die Cbronik von vor 1777 anknüpfte.

In mehrfacher Hinsicht ist Schubart seinem Landsmann Wieland - von dem er sich auf andere Weise sehr früh distanziert hat - und der Verkörperung dessen, was man in 
Deutschland die „idealistische Reaktion“ nennt, sehr nahe. Die Exzesse, die in den ersten Monaten begangen wurden, zeigten für Schubart, daß jede Bewegung dieser Art wenn nicht eine entscheidende Veränderung in den Herzen vor sich gehe - der Gefahr ausgesetzt sei, mißbraucht zu werden und in blutige Anarchie zu degenerieren. Ebenso behart die Cbronik häufig auf der entscheidenden Unterscheidung zwischen „falscher Aufklärung“ und ,wahrer Aufklärung“ - diese ist in der in Deutschland üblichen Weise zu verstehen. Die Deutschen hätten nach Schubart das Glück, durch weise und um das Wohlergehen ihrer Untertanen besorgte Fürsten regiert zu werden; deshalb bräuchten sie keinen Umsturz. Schubart nennt diese mustergültigen Souveräne, unter denen Karl Eugen demonstrativ hervorgehoben wird.

Wie viele seiner Landsleute wünschte Schubart, daß es zu einem harmonischen Einvernehmen zwischen Souverän und Volk kommen möge. Sein Traum war die konstitutionelle Monarchie: eine Mischung aus britischem Herrschaftssystem, Schweizer Republik und aufgeklärten Regierungen, wie sie in Deutschland existierten. Die Freiheit sei dort nicht ein Ferment der Zwietracht, sondern ein Faktor der Harmonie. Einen Moment erschien ihm diese idyllische Hoffnung zum Greifen nahe: Das Föderationsfest in Straßburg wie das in Paris bestätigten diese optimistische Vision. Schubart begrüßte darin das, was Michelet „un immense élan de concorde au bout d'un siècle de disputes" nannte. Der Entwurf der Konstitution, den die Chronik breit analysierte, glich einer „monarchie temperée“, in der der Hydra der Anarchie die Zügel angelegt sind.

Aber die übrigen Vorzeichen waren beunruhigender: der "Dämon der Agitation“ könnte leicht den Sieg davontragen. Schubart brandmarkte die Zügellosigkeit und begann sogar, Argumente beizubringen, die E. Burke anerkennen sollte. Ebenso brandmarkte er T. Müntzer und gab Luther recht, ersteren hart verurteilt zu haben. Angesichts der Ereignisse, die Schritt für Schritt gleichzeitig beide Interpretationen bestätigten, zögerte Schubart: der Pariser Pöbel gab das Beispiel der Anarchie, während Straßburg ein ermutigenderes Beispiel bot.

Sechsundzwanzig Monate schwankte die Chronik also zwischen Enthusiasmus und Skeptizismus, zwischen Hoffnung und Pessimismus - je nach den Gefühlen Schubarts. Aber seine Vorbehalte, selbst wenn sie sich in extremer Form darstellen, implizieren nicht eine Verurteilung der Prinzipien, die die revolutionäre Bewegung für sich reklamierte. Schubarts plötzlicher Tod im September 1791 erlaubte ihm nicht mehr, die Ereignisse mitzuerleben, die viele Deutsche dazu brachten, eine radikalere, oft ablehnende Position einzunehmen.

\section{Diskussion zum Referat von Jean Clédière}

\section{Leiter: Eberbard Weis}

Eberbard Weis leitete die Aussprache mit einem Vergleich zwischen Schubart und Pestalozzi ein. Er hielt Schubarts Haltung gegenüber der Französischen Revolution für sehr viel typischer für die deutschen Intellektuellen als diejenige Pestalozzis, wobei die Zweifel der Intellektuellen an der Französischen Revolution erst 1792/93 einge- 
setzt hätten, Schubart aber, der bereits 1791 gestorben sei, diese schon viel früher geäußert habe. In diesem Zusammenhang wies $M$. Botzenbart auf ein methodisches Problem hin. Er fragte, inwieweit die Texte der Autoren auf die Zensur abgestellt, also "taktisch" verfaßt gewesen seien. Nach $J$. Clédière habe es sich allerdings bei Schubart mehr um persönliche Vorsicht als um Taktik gehandelt, da Herzog Karl Eugen von Württemberg selbst eine sehr zweideutige Haltung zur Revolution gehabt hatte, die den Autor zu doppelter Vorsicht gezwungen hätte. Für den Historiker bedeute dies, daß sich aus Schubarts Schriften kein System herauskristallisieren lasse.

Die Bedeutung der Presse im allgemeinen (seit 1770) unterstrich J. Voss. Ohne Presse hätte es im Reich keine Informationsmöglichkeiten über die Revolution in Frankreich gegeben, die sie treffende Zensurpraxis wäre jedoch in den verschiedenen deutschen Staaten sehr unterschiedlich gewesen. Hierzu präzisierte $W$. Siemann, daß sich 1792, dem eigentlichen "Schwellenjahr" des Pressewesens, die Zensur auf die Presse eingestellt habe und Pressezensurbehörden geschaffen worden seien. So zeigten die Beispiele Baden, Österreich und Württemberg, daß die Zeitungen als Problem begriffen worden seien. In Württemberg habe man die Institutionalisierung der Zensur damit erklärt, daß man die „wahre“ Aufklärung gegenüber der „falschen“ fördern wolle. Auch der Referent unterstrich die großen Unterschiede in der Zensurpraxis, die den Autoren die Möglichkeit gegeben hätten, allein durch einen Ortswechsel moderatere Regelungen zur Publikation zu nutzen.

Im Mittelpunkt der Diskussion stand schließlich das Bild, das Schubart vom Dritten Stand im Frankreich der Revolution hatte. J. Laspougeas erklärte hierzu, Schubart habe zwar von einer Versöhnung unter den Franzosen im Anschluß an die Ereignisse von 1789 gesprochen, nicht aber die Krise von 1791 erkannt, in deren Verlauf der Dritte Stand zerbrochen sei. Daran schloß sich die Frage an, wie Schubart über die Trennung von Aktiv- und Passivbürgern gedacht und wie er die Zivilkonstitution des Klerus eingeschätzt habe. Der Referent zeigte nun, daß Schubart tatsächlich diese Krise nicht erkannt habe, über die Einschränkung des Wahlrechts nichts sage und zur Zivilkonstitution nur ein oder zwei Anspielungen mache. Clédière führte dies auf ein Informationsdefizit zurück, das dem Autor lediglich ein unmittelbares Reagieren auf die Ereignisse erlaubt habe. Er habe einen politischen Kopf besessen, der detailliert lediglich die Konstitutionstexte habe kommentieren können. Außerdem habe er eine tiefe Feindschaft gegen die Jesuiten gehegt, die er für seine Einkerkerung vọr der Revolution verantwortlich gemacht habe.

Auf die Haltung der Protestanten gegenüber der Zivilkonstitution gingen schließlich noch P.-A. Bois und J. Tulard ein. P.-A. Bois zeigte, daß die deutschen Protestanten zwar die Dechristianisierungspolitik in Frankreich verurteilt, die Zivilkonstitution aber mit Interesse studiert haben. Diese Beobachtung bestätigte $J$. Tulard auch für die französischen Protestanten. Er fügte noch hinzu, die antireligiöse Politik habe auch die Juden getroffen, deren Synagogen geschlossen worden seien. 
